\title{
Development of a Fiber-Optic Sensing System for Train Vibration and Train Weight Measurements in Hong Kong
}

\author{
C. C. Lai, ${ }^{1}$ Jacob C. P. Kam, ${ }^{2}$ David C. C. Leung, ${ }^{2}$ Tony K. Y. Lee, ${ }^{2}$ Aiken Y. M. Tam, ${ }^{2}$ \\ S. L. Ho, ${ }^{1}$ H. Y. Tam, ${ }^{1}$ and Michael S. Y. Liu ${ }^{1}$ \\ ${ }^{1}$ Department of Electrical Engineering, Hong Kong Polytechnic University, Kowloon, Hong Kong \\ ${ }^{2}$ Mass Transit Railway, Fo Tan Railway House, Fo Tan, Shatin, NT, Hong Kong \\ Correspondence should be addressed to Tony K. Y. Lee, tkylee@mtr.com.hk
}

Received 3 April 2012; Accepted 29 May 2012

Academic Editor: Yu-Lung Lo

Copyright ( 92012 C. C. Lai et al. This is an open access article distributed under the Creative Commons Attribution License, which permits unrestricted use, distribution, and reproduction in any medium, provided the original work is properly cited.

A novel operation system to detect train vibration and train weight using FBG sensing network has been designed and tested in Hong Kong. The purpose of the system is for real time condition monitoring of trains. Because of the fast response of optical systems, the trains can be monitored in real-time during its normal service without any special arrangement. Hence, the condition checking can be realized without any disruption on the operating condition of the railway system.

\section{Introduction}

The health condition monitoring of trains is becoming increasingly important in modern cities like Hong Kong that rely heavily on mass transportation systems. Due to the heavy reliance of the general public on public transportation, it is inconceivable to have long delays in any of the public transportation systems, not to mention the suspension of services for many days. In the past, it is common to use the conventional approach of planning the maintenance work using either time-interval-based or mileage-based scheduling. However, it is well known that not all systems are exactly the same and hence the queuing approach may not be the best arrangement for the railway industry. In other words, there may be trains which should be serviced earlier than being planned, and there are also trains which do not need to be looked at even though they have been scheduled for routine checkup. To make the best use of the limited maintenance resources available, a reliable train health conditioning monitoring system is crucial.

This paper describes a novel health monitoring system using optical fibres. The benefit of optical fiber sensor is electromagnetic immunity (EMI), reliability, and durability. Indeed EMI is crucial because it is common to have $25 \mathrm{kV}$ overhead lines in mainline trains and there are also hundreds, if not thousands of amperes of current flowing in the return rail. The use of optical fiber as both the sensing element and the transmission media can minimize the interference of electromagnetic waves from the traction motors and from the power lines of mainline systems.

The optical sensor being exploited in this study is based on fibre Bragg grating (FBG) technology [1-4]. The FBG sensor network is installed on a $2.5 \mathrm{~km}$ long track in Hong Kong and it is close to the Siu Ho Wan depot (SHD) between Tung Chung Station and Yam O station in the Tung Chung Line.

To validate the measured data, a trainset "TCL 08 " with cars carrying sand bags with known weights was tested at around 11:41:42.6 am on 20th September, 2012. The data being recorded are then analysed offline carefully. An antiderailment system based on the calibrated results as described in this paper are now in operation.

\section{Fibre Bragg Grating Sensors Network}

Fiber Bragg grating (FBG) is an optical sensor in an optical fiber for sensing changes in either strain or temperature. FBG sensor has been selected for the study being carried out 


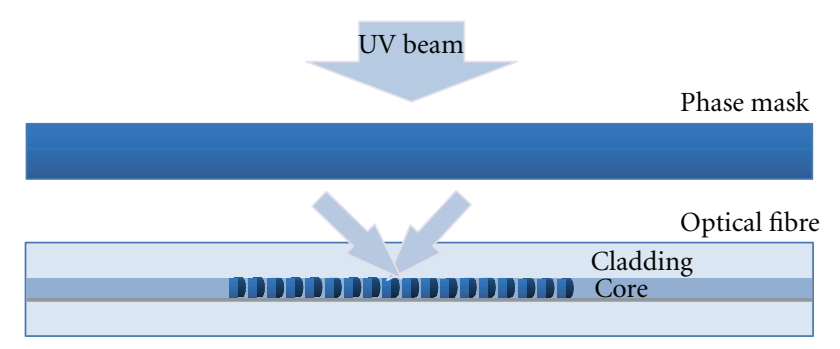

FIGURE 1: Fabrication of optical sensors using Ultraviolet lights and masks.

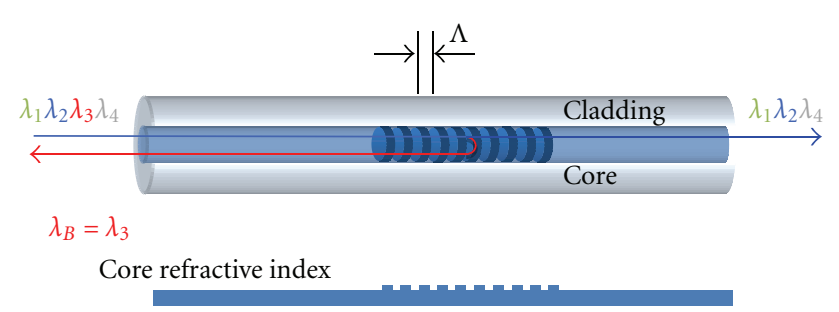

Figure 2: Changes in core reflective index after FBG has been inscribed on the optical fibre.

because of its fast and quick time response as well as its EMI property.

2.1. The Working Principle of FBG. An Fibre Bragg Grating (FBG) sensor is an optical device that measures strains by means of detecting changes in the reflected wavelength of light. A FBG sensor can be fabricated on a short-length $(\approx 1 \mathrm{~cm})$ of single-mode optical fibre, which is as thin as human hair, by exposing the sensors to ultraviolet light through a phase mask [5] as shown in Figure 1.

The FBG sensor consists of a short length of periodic refractive-index [6] changes as shown in Figure 2 inside the optical fibre. A light source is used to pass a band of light spectrum into the sending end of the optical fibre. Without the FBG sensor, the light passes through the optical fibre unobstructed. When there is an FBG sensor, a narrowband of wavelength of the light spectrum is reflected back to the sending end and these reflected wavelengths are analyzed by an optical interrogator. The light being reflected back has a spectrum that characterizes the pitch (e.g., the separation between two periodic marks) of the periodic refractiveindex variation. It can be seen in Figure 2 that if the input light with a band of wavelengths $1,2,3$, and 4 is fed into the optical fibre, it is only wavelength $B=3$ which will be reflected back because the grating satisfies the Bragg condition of $B=2 n \Lambda$, where $B$ is the Bragg wavelength, $n$ is the refractive index of the core material, $\Lambda$ is the pitch of the grating which is defined by the phase mask during FBG inscription. The rest of the wavelengths 1,2 , and 4 are transmitted through the grating. As the pitch is changed when the FBG is subjected to strain (i.e., the pitch becomes longer, when the FBG sensor is being extended, and becomes shorter when the FBG sensor is being compressed), there will be corresponding changes in the reflected wavelength.

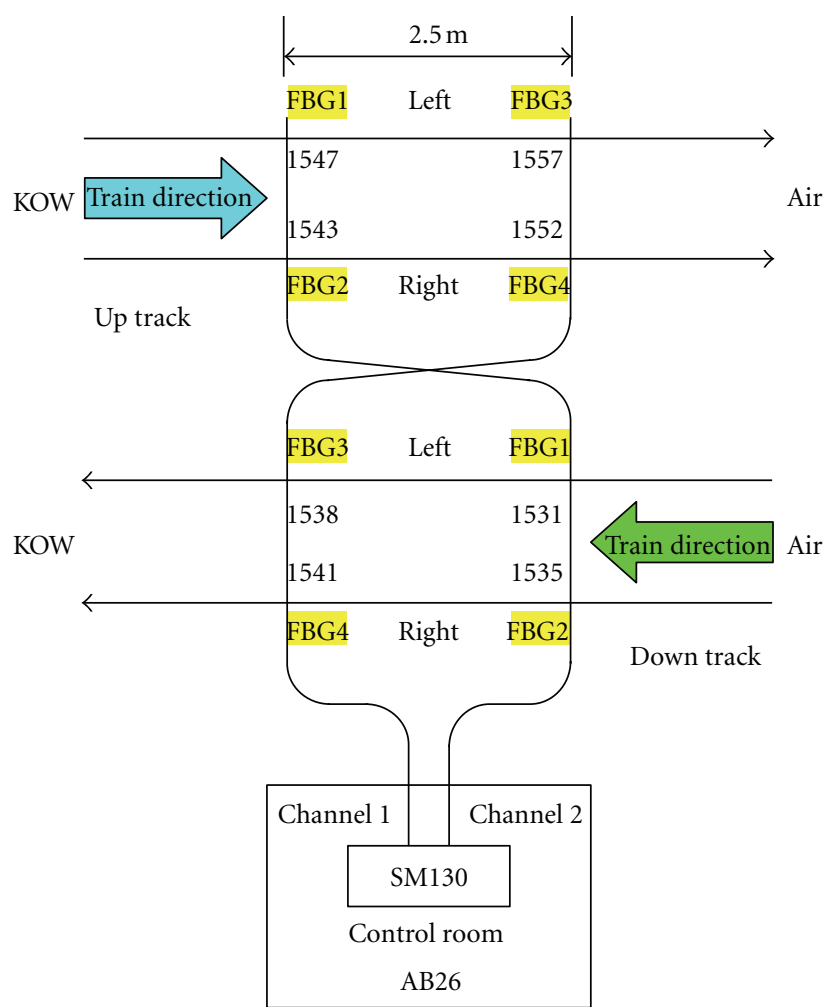

Figure 3: Schematic of Train Load balance index monitoring system in SHD. The sensor group 1543, 1547, 1552, 1557 are installed on up track while sensor group 1531, 1535, 1538, 1540 are installed on down track.

Since the parameter of measurement is the wavelength of light which is not affected by electromagnetic fields, the process is immune to electromagnetic interference and hence is intrinsically more stable than any electrical monitoring system in an electr4magnetically noisy environment which is typical in an electrified railway.

2.2. FBG Sensor Network. The FBG sensor network being installed includes a number of FBGs in an optical fiber. The length of the optical fibre can be as long as $100 \mathrm{~km}$ and many FBGs with different grating characteristics can be inscribed on the same optical fibre which is connected to a commercially available interrogator system as shown in Figure 3 in the control room. This interrogator system is further connected to a computer with an application program which is developed in-house for train vibration monitoring and train weighing.

Figure 4 shows a typical pickup from a sensor installed on the track with the passage of a 12-car train. It is noted that there are 4 wheels in one car and hence the first 4 peaks on the left-hand side of the upper figure correspond to the first car. It can be seen that there are more vibrations in Cars 6 and 8 (when counting from the left hand side) and such observation would allow the engineer to pay attention to cars with abnormal or excessive vibrations. Subsequent investigation revealed that there were roundness problems in 


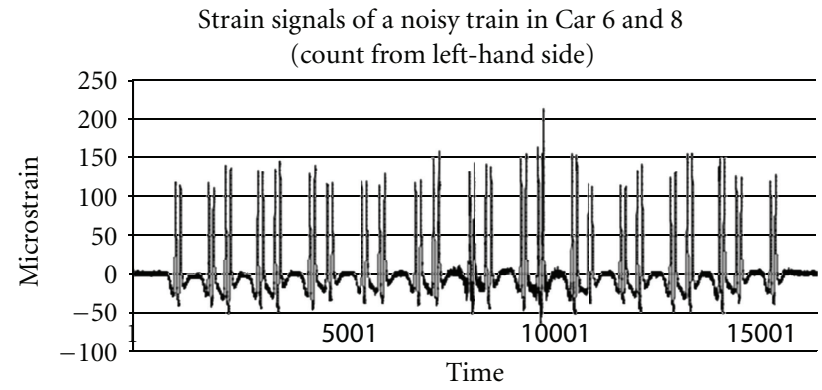

(a)

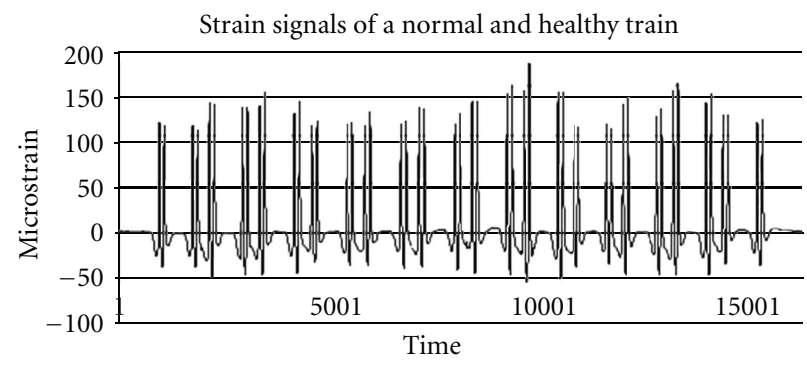

(b)

FIGURE 4: Strains picked up by optical sensors mounted on the track with the passage of a 12-car train.

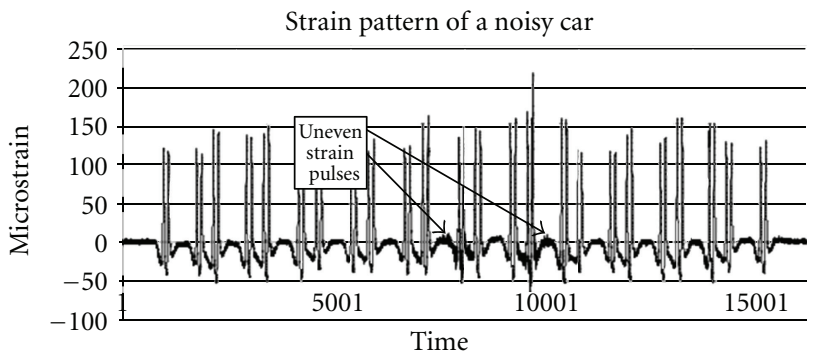

Figure 5: Strains picked up on the track due to the passage of a noisy train.

the wheels of Cars 6 and 8 and this will be described in more details in subsequent sections of this paper.

\section{Applications}

3.1. Monitoring of Imperfections in Train Wheels. With the installation of the sensors on the tracks, a wealth of investigations could be carried out readily. For example, from the strain measurements at the track, it was confirmed that there were some noisy trains. It was suspected that these noisy trains might have relatively imperfect wheels. Hence, a series of tests were carried out. The first test is to identify a noisy train (a train producing strains as shown similar to the upper half of Figure 4) using an FBG sensor installed on the rail track as described in the previous section.

The principle of wheel imperfection detection by FBG strain [4] sensors installed at track is based on the fact that wheel defects such as flange pits, wheel flats, and particularly out-of-round wheels which are also known as polygonal wheels, will exert periodic impact force on the track. In this work, it was found that an imperfect wheel will produce an uneven strain impulse on the track. In contrary, a newly turned wheel will produce a symmetrical strain impulse. In another approach, one attempts to relate the interaxial vibrations as shown in Figure 5 with a wheel that has outof-roundness. In order to compare parameters from two different systems, the interaxial vibrations were quantified by a vibration index which is obtained arithmetically by considering the train speed, vibration frequency, and magnitude as a whole (Figure 6).
The detail derivation of the vibration index, is however, a proprietary information of the authors and hence cannot be described in too much details in this paper. Figure 7 shows the vibration indexes obtained from a noisy train.

The local vibrations such as those in Cars 6 and 8 (counting from the left) of Figure 7 have a vibration index of $1.5+$ which are higher than those of the other cars in the same train with a vibration index lower than 1.1.

The wheel of the train cars with out-of-roundness (Cars number 6 and 8) [7] are measured.

The wheels on Car number 6 were turned right after the out-of-roundness measurement while the wheels on Car number 8 were kept unturned as a control. The wheels outof-roundness after turning were between 0.05 to $0.07 \mathrm{~mm}$. The strain pattern and vibration indexes of the serviced train are shown in Figure 8 from which one can observe that the vibration at Car number 6 has been eliminated after wheel turning while the vibration at Car number 8 persists. By comparing Figure 7 with Figure 9, it can be seen that the vibration index of Car number 8 (unturned wheels) remained high at around 1.9 while the index of Car number 6 (turned wheels) was greatly reduced from 1.8 to 0.8 . This shows that the vibration index, which is deduced from the FBG strain sensor measurement results, is an effective means to distinguish wheels with out-of-roundness from wheels which are healthy.

3.2. Train Weight Measurement. The Bragg wavelengths of the FBGs will change because of deformation of the rail when the train passes over the location at which the FBG is installed. The data are being logged by a computer with a sampling frequency of $1000 \mathrm{~Hz}$. The waveforms are shown as in Figure 10 below.

At the initial stage of the study, it was found intuitively that the amplitude of the peaks such as $P 1$ as shown in Figure 10 is broadly corresponding to the weight of the axle. However, it is noted that the amplitude of the peak can be found in different ways. Therefore, four approaches are proposed as given below. Here, $W 1$ and $W 2$ are defined as the respective weight of axles 1 and 2 of the same bogie. $P 1$ and $P 2$ are the peaks of the signal excited by the load being applied to axle 1 and axle 2 respectively. $V 1, V 2$, and $V 3$ are the respective valleys which are generated when the train car 


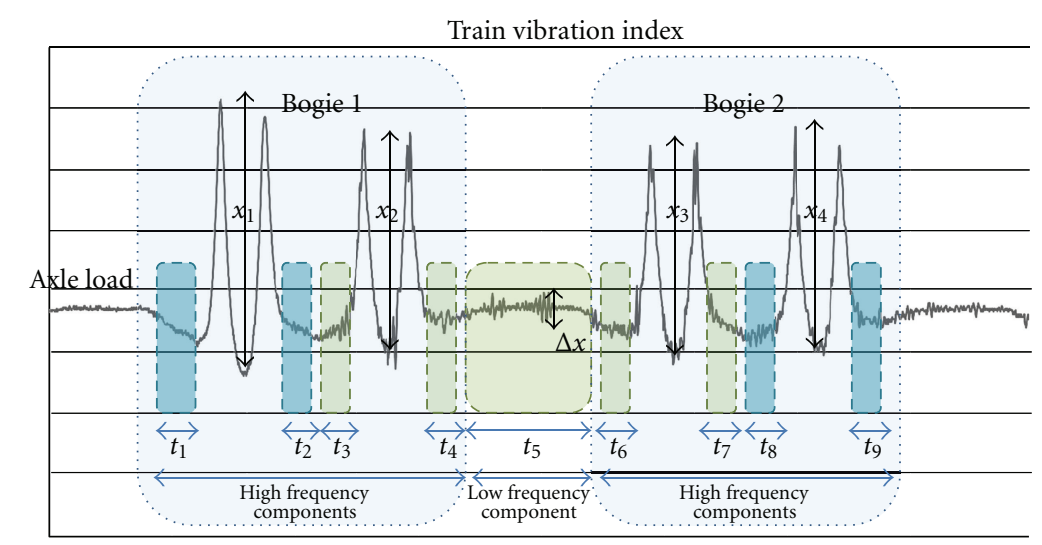

Time

Vibration index $=\sum_{n=1 \ldots 9}^{n} K_{n} f\left(\Delta X_{n} / X_{n}, t_{n}\right)$, where $K_{n}$ is the weighting factor of each component

FIGURE 6: Graphical explanation of how the vibration index is being derived after the vibration waveforms are sectionalized and weighted in order to generate the vibration index.

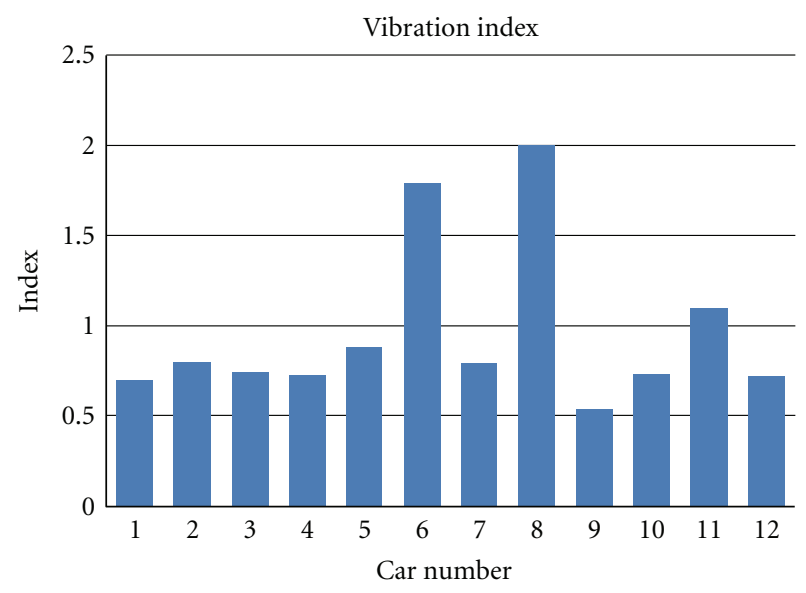

FIGURE 7: Vibration index of the 12 cars.

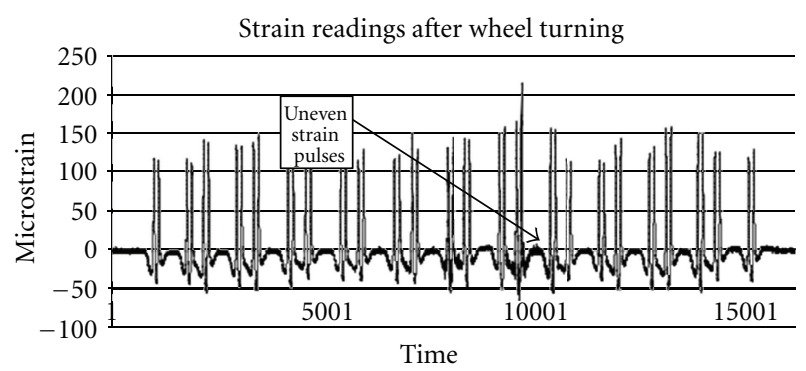

FIGURE 8: The strain readings after wheel turning.

has moved away from the rail. The weight of the axle can then be found from the four approaches as given below:

Approach 1: $W 1=(P 1-V 1) ; W 2=(P 2-V 2)$, Approach 2: $W 1=(P 1-V 2) ; W 2=(P 2-V 3)$,

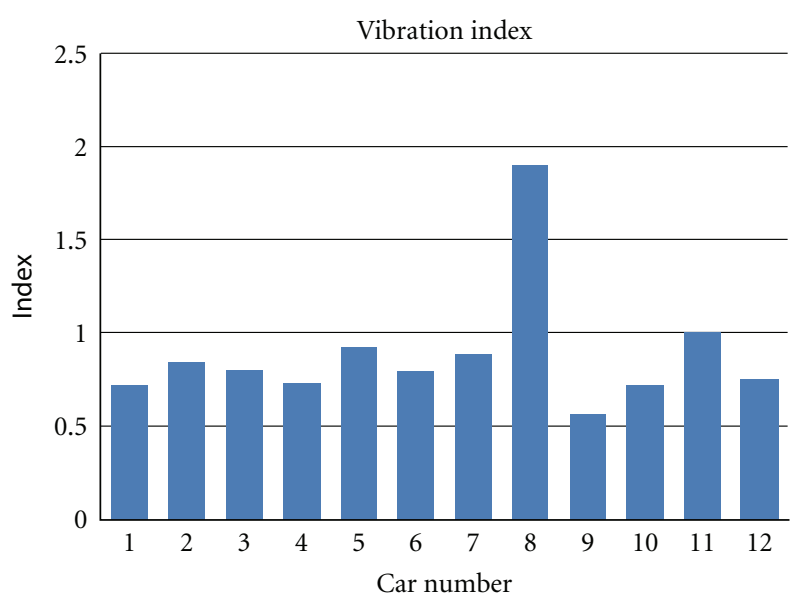

FIGURE 9: Vibration index of the train with the wheels of car 10 turned.

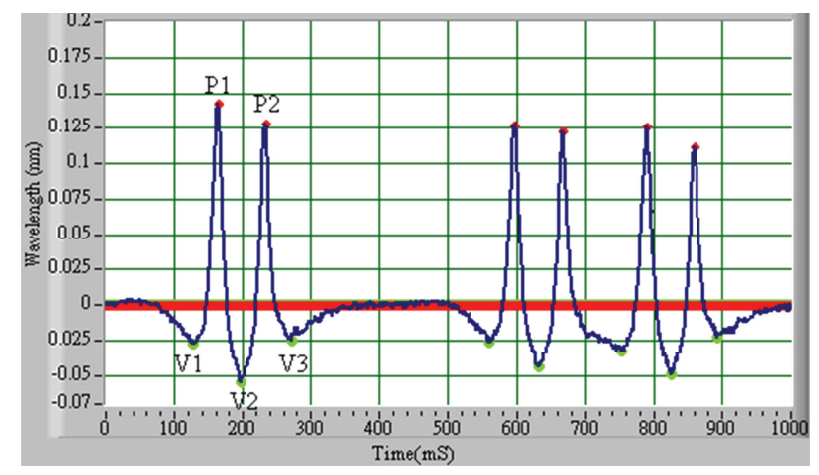

FIGURE 10: Strain measurement using FBG when a wheel of a train is passing through. 


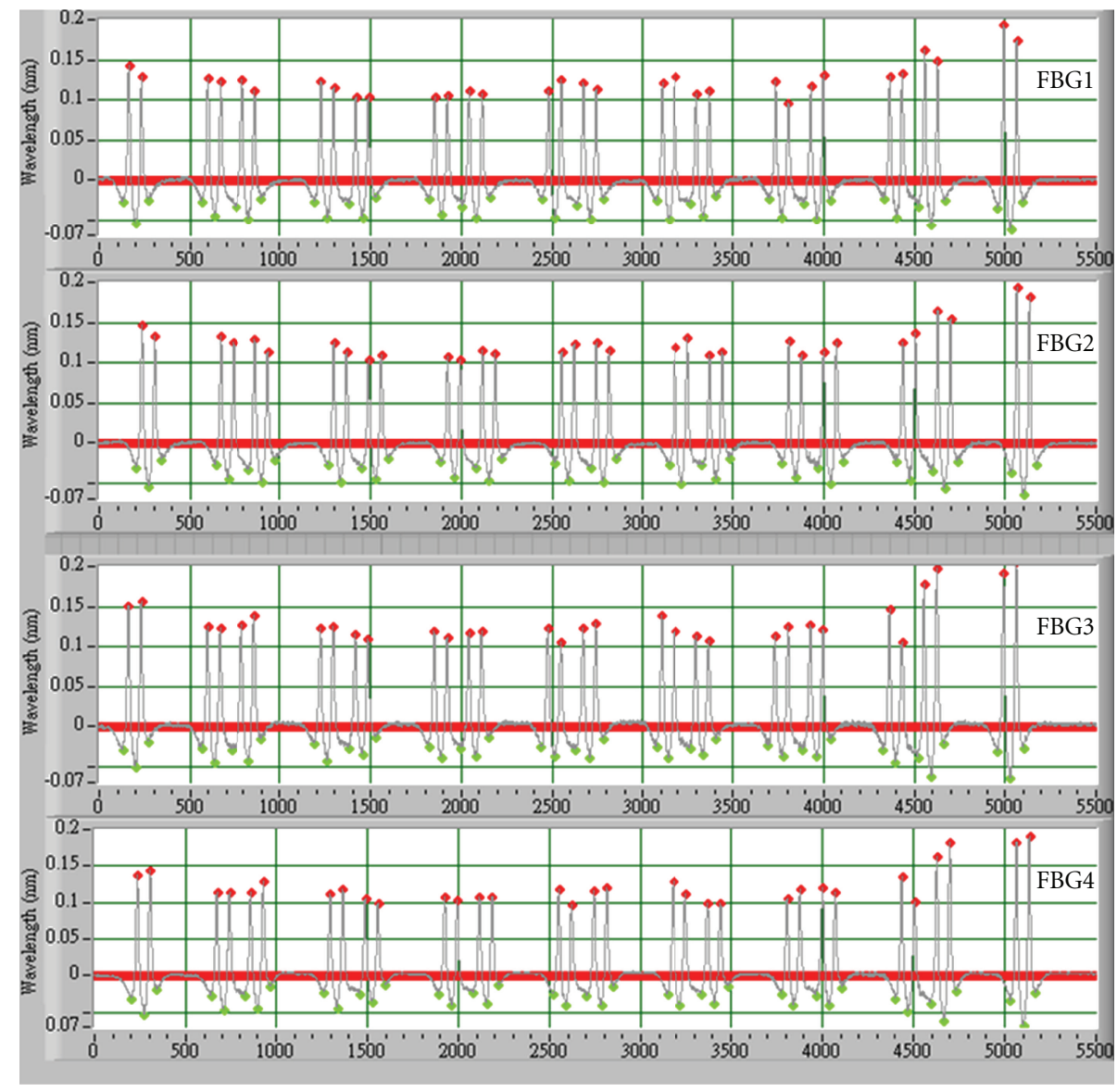

FIGURE 11: Wavelength signals of the trainset "TCL 08 " as measured in the down track.

Approach 3: $W 1=(P 1-(V 1+V 2) / 2) ; W 2=(P 2-$ $(V 2+V 3) / 2)$,

Approach 4: $W 1=P 1 ; W 2=P 2$.

3.2.1. The Static Weight Data. The trainset "TCL 08" with the weights of all the wheels were measured and the static data are then used to compare with the strain measured by using FBGs.

3.2.2. Measurement Results. The wavelengths signal of the trainset "TCL 08" are shown as in Figure 11. These signals are measured in the down track.

The strains as derived from the shifts in wavelengths are then feed to the application program and then compared with static weight data. In order to calibrate the system, the static weight of one axle was equated to the amplitude of the FBG signal when the train was moving very slowly over the track at which an FBG sensor was installed. The ratio, which is equal to the weight of axle/amplitude of the FBG signal, is then used to convert the dynamic amplitude of each FBG signal in train weight. The results of the four approaches in finding the amplitude of the FBG signals are as shown in Table 1. The best scenario is Approach 4 which has the minimum error percentage. The average error from the four FBGs are $10.39 \%$ and, bearing in mind that the "dynamic" weight of trains is normally considered to be $10 \%$ higher than their static weight, the measured data are considered to be in excellent agreement with the static data.

3.3. Train Antiderailment System. One of the most important safety assessments for running train on rail is on the possibility of derailment, particularly in negotiating curve and track twist. The likelihood of derailment is very much dependent on the conditioning of track and the vehicle. The former refers to the deviation of the maintenance limits on the track geometries, while the latter refers to the conditions of the vehicle suspensions system together with their wheel profiles. Checking of both conditions will have to be done during off-traffic hours under prescheduled intervals, unless there are means for online in-service checking.

Derailment caused by track twist is related to the combination of the horizontal guiding force and the reduction of the vertical wheel-load of the leading wheel. The resultant of the two force vectors may cause the guiding wheel to climb the rail.

Nadal's limit $(Y / Q)$ on the lateral force $(Y)$ on the wheel being evaluated for derailment and its vertical load $(Q)$ is used to measure the probability of flange climbing. It has to be kept to an acceptable limit. Figure 12 indicates the relationship of the forces at the wheel/rail interface. 
TABLE 1: The result of the four approaches.

\begin{tabular}{lcccc}
\hline $\begin{array}{l}\text { Up track } \\
\text { Error \% }\end{array}$ & Approach 1 & Approach 2 & Approach 3 & Approach 4 \\
\hline FBG 1,2 & 11.51 & 12.51 & 14.39 & 12.48 \\
FBG 3,4 & 11.53 & 13.10 & 12.59 & 9.94 \\
\hline Down track & & & & \\
Error \% & Approach 1 & Approach 2 & Approach 3 & Approach 4 \\
\hline FBG 1,2 & 14.43 & 14.37 & 12.00 & 9.40 \\
FBG 3,4 & 12.36 & 12.82 & 12.34 & 9.74 \\
\hline
\end{tabular}

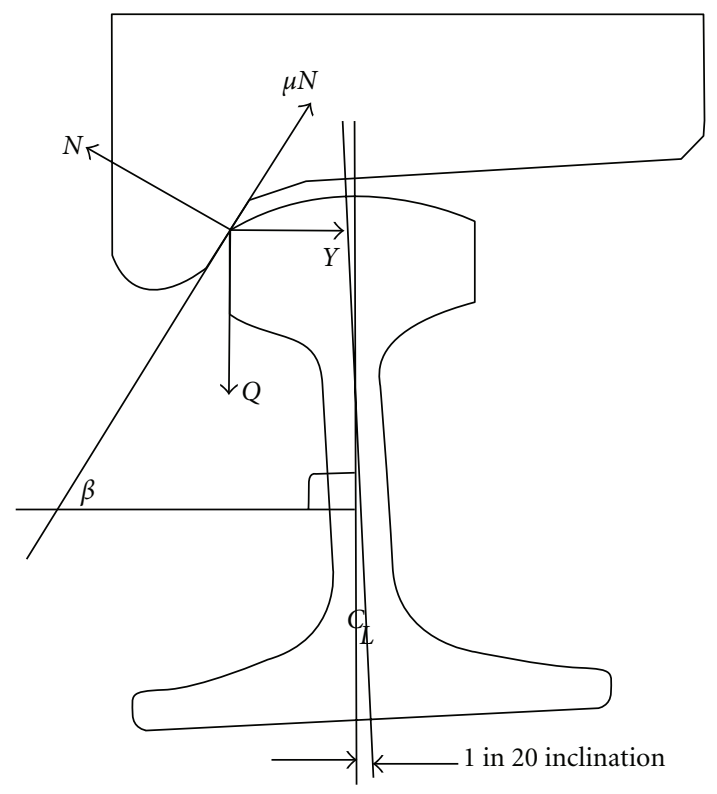

$\frac{Y}{Q}=\frac{\tan \beta-\mu}{1+\tan \beta}$,

where

$Y$ : lateral force at wheel angle

$Q$ : vertical load on the wheel

$\beta$ : wheel ange angle at plane of contact

$\mu$ : coefficient of friction at ange/rail contact point

FIGURE 12: Relationship of forces at the wheel/rail interface.

As lateral force [8] is inevitably present for train wheels in negotiating track curve and twist, it is important to ensure that the wheel sets must be designed to operate with adequate vertical loads to keep the Y/Q ratio to fall within limits.

Load transfer between wheel sets is thus critical and must be controlled also to within a reasonable limit, which is no more than $60 \%$ of the load from one wheel being transferred to the other as shown in (1), where $Q_{1}$ and $Q_{2}$ are the vertical loads of two wheels of the same axle acting on rail:

$$
\text { off-loading ratio } \frac{\Delta Q}{Q}=\frac{\left(Q_{1}-Q_{2}\right)}{\left(Q_{1}+Q_{2}\right)}<0.6 \text {. }
$$

The weight of the train acting on the rail by the wheel would create both tensile strain and stress on the rail beam. By putting FBG on rail, their corresponding changes in $\Lambda$ can be detected readily. Practically, changes in the FBG grating $\Lambda$ sensed would be presented as changes in $\lambda$ and, hence, according to the principle described, the weight of the train will trigger a shape change in wavelength $(\Delta \lambda)$. Once it is captured, it will represent the weight of the wheel acting on the rail $Q$.

As the off-loading ratio $\Delta Q / Q$ concerns only on the relativity ratio of the loadings and not on the absolute value of the vehicle weights, thus, the temperature effect and the need of fine tuning and resolving the transfer function of the $\Delta \lambda$ with respect to the weight $Q$ are not a matter of concern as they all will be canceling out in deriving the off-loading ratio $\Delta Q / Q$.

It is noted that because one is more concerned about the ratio of the axle weight on the two sides of the axle when determining the potential derailment danger, there is no real need to measure the axle weight very accurately. The fact that the sensors were measuring the axle weight very accurately is, therefore, an additional bonus which might provide further intelligence into the development of an even more comprehensive smart railway conditioning monitoring system in future.

\section{Summary}

An optical system using Fibre Brag Grating has been designed and tested in a commercial railway testbed. Electromagnetic interferences could cause serious problems in main line railways operating with a $25 \mathrm{kV}$ supply. Hence, this research is focused on the use of optical sensors which are immune of EMI. In essence, this study reports the use of FBG sensors for detecting vibrations from noisy trains as well as train weigh, thereby paving the way for the development of an antiderailment system which is an essential requirement for a modern railway.

The response signal obtained by the FBG sensors is positive and clear enough for further development. Apart from the objectives mentioned above, the FBG system could be tailor made for many additional railway applications including the following:

(a) counting the numbers of axles in and out of a given section (track circuit occupation), that is, for axle counter,

(b) train identification by tracking the differences of axle bases, weights, and car numbers of various train stocks,

(c) speed detection.

In summary, a small piece of FBG sensor can generate lots of train running information with good data integrity. It can be applied to monitor the train running performances of the railway by building a virtual system on top of the conventional ones as a standby system both on operational and safety aspects.

Particularly, FBG axle counter system can be built on top of existing conventional axle counter system of a moving block signal system to allow a virtual track circuit to remain operational in case of emergency or for running engineering train during nontraffic hours.

Further analysis and measurements together with a fully blown development on its mounting on rail is necessary 
before the maturity of the FBG sensor technology can be put into practical usage in the highly conservative railway industry.

\section{Acknowledgments}

The authors would like to acknowledge the support from the MTR for allowing a number of tests to be carried out in an operational rail line.

\section{References}

[1] P. F. Weston, C. J. Goodman, P. Li, C. S. Ling, R. M. Goodall, and C. Roberts, "Track and vehicle condition monitoring during normal operation using reduced sensor sets," Transactions Hong Kong Institution of Engineers, vol. 13, no. 1, pp. 47-53, 2006, Special Issue on Railway Development in the 21st Century.

[2] K. Y. Lee, K. K. Lee, and S. L. Ho, "Exploration of using FBG sensor for axle counter in railway engineering," WSEAS Transactions on Systems, vol. 3, no. 6, pp. 2440-2447, 2004.

[3] K. K. Lee and S. L. Ho, "Unconventional method of train detection using fibre optic sensors," Transactions Hong Kong Institution of Engineers, pp. 16-21, 2006, Special Issue on Railway Development in the 21st Century.

[4] A. D. Kersey, M. A. Davis, H. J. Patrick et al., "Fiber grating sensors," Journal of Lightwave Technology, vol. 15, no. 8, pp. 1442-1462, 1997.

[5] K. O. Hill, B. Malo, F. Bilodeau, D. C. Johnson, and J. Albert, "Bragg gratings fabricated in monomode photosensitive optical fiber by UV exposure through a phase mask," Applied Physics Letters, vol. 62, no. 10, pp. 1035-1037, 1993.

[6] T. Erdogan, "Fiber grating spectra," Journal of Lightwave Technology, vol. 15, no. 8, pp. 1277-1294, 1997.

[7] A. Johansson, "Out-of-round railway wheels-assessment of wheel tread irregularities in train traffic," Journal of Sound and Vibration, vol. 293, no. 3-5, pp. 795-806, 2006.

[8] P. Remington and J. Webb, "Estimation of wheel/rail interaction forces in the contact area due to roughness," Journal of Sound and Vibration, vol. 193, no. 1, pp. 83-102, 1996. 

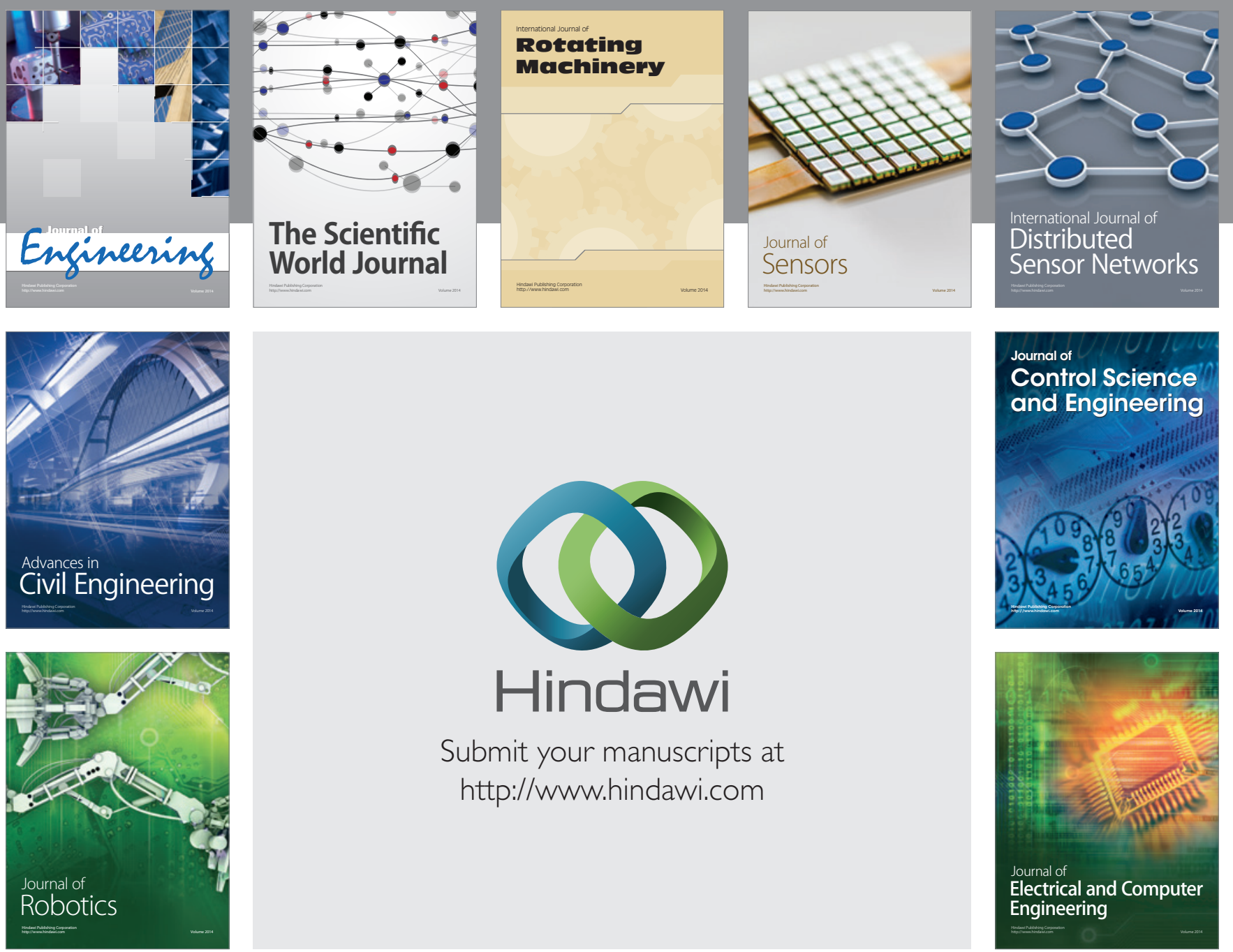

Submit your manuscripts at

http://www.hindawi.com
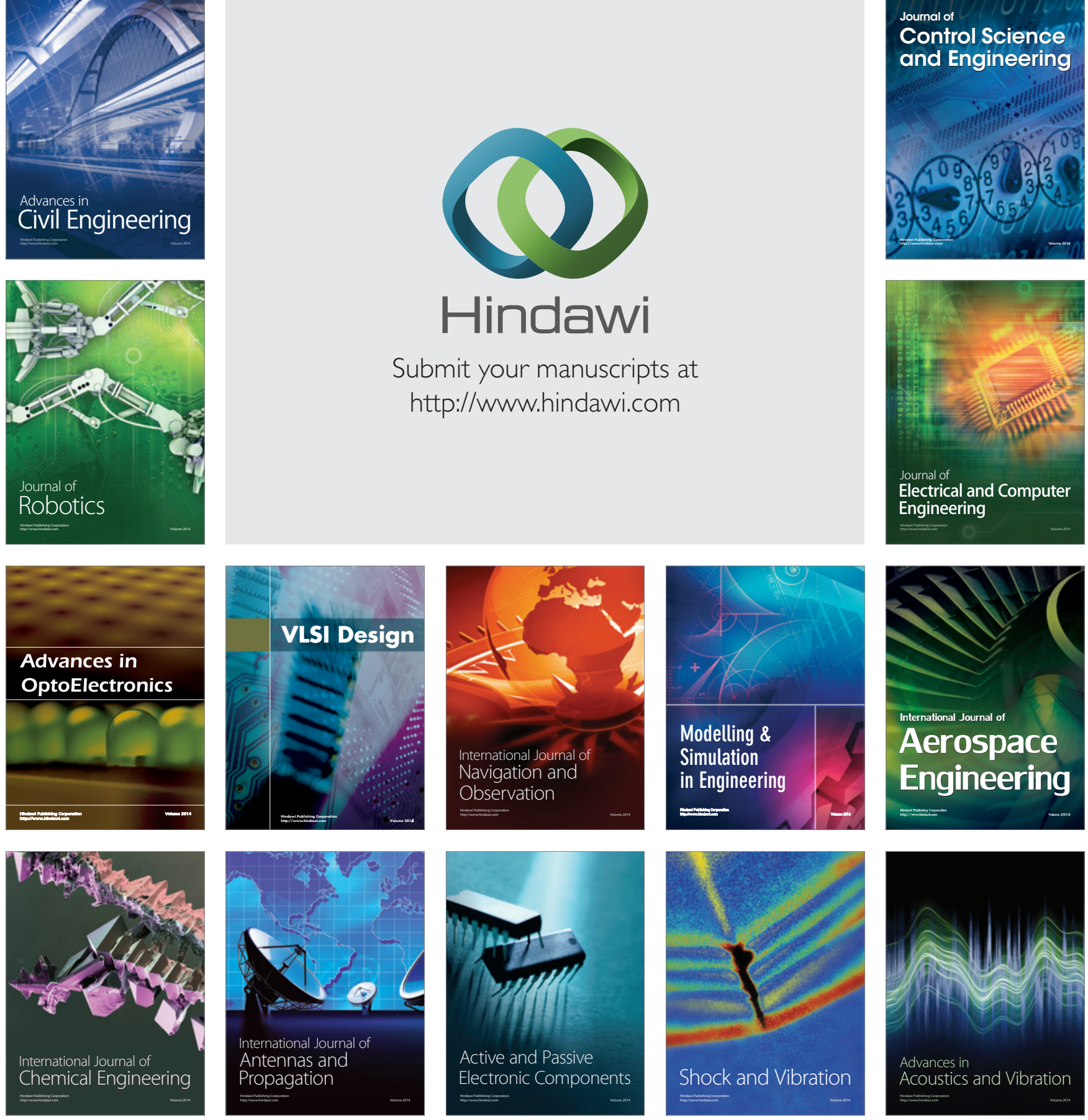